\title{
Prevalence and population structure of Staphylococcus aureus nasal carriage in healthcare workers in a general population. The Tromsø Staph and Skin Study
}

\author{
K. OLSEN ${ }^{1,2 *}$, M. SANGVIK ${ }^{3}$, G. S. SIMONSEN ${ }^{1,3}$, J. U. E. SOLLID ${ }^{3}$, \\ A. SUNDSFJORD ${ }^{3}$, I. THUNE ${ }^{2,4}$ AND A.-S. FURBER G ${ }^{1,2}$ \\ ${ }^{1}$ Department of Microbiology and Infection Control, University Hospital of North Norway, Tromso, Norway \\ ${ }^{2}$ Department of Community Medicine, Faculty of Health Sciences, University of Tromsø, Tromsø, Norway \\ ${ }^{3}$ Department of Medical Biology, Faculty of Health Sciences, University of Tromsø, Tromsø, Norway \\ ${ }^{4}$ Department of Oncology, Oslo University Hospital, Ullevål, Oslo, Norway
}

Received 21 December 2011; Final revision 21 February 2012; Accepted 21 February 2012; first published online 22 March 2012

\section{SUMMARY}

Healthcare workers (HCWs) may be a reservoir for Staphylococcus aureus transmission to patients. We examined whether HCW status is associated with $S$. aureus nasal carriage and population structure (spa types) in 1302 women (334 HCWs) and 977 men ( $71 \mathrm{HCWs)} \mathrm{aged}$ 30-69 years participating in the population-based Tromsø Study in 2007-2008. Multivariable logistic regression models were used. While no methicillin-resistant $S$. aureus (MRSA) was isolated, overall, $26 \cdot 2 \%$ of HCWs and $26 \cdot 0 \%$ of non-HCWs were $S$. aureus nasal carriers. For women overall and women residing with children, the odds ratios for nasal carriage were 1.54 [95\% confidence interval (CI) $1 \cdot 09-2 \cdot 19$ ] and $1 \cdot 86$ (95\% CI 1.14-3.04), respectively, in HCWs compared to non-HCWs. Moreover, HCWs $v s$. non-HCWs had a 2.17 and 3.16 times higher risk of spa types t012 and t015, respectively. This supports the view that HCWs have an increased risk of $S$. aureus nasal carriage depending on gender, family status and spa type.

Key words: Healthcare worker, infection control, population-based, residing with children, Staphylococcus aureus nasal carriage.

\section{INTRODUCTION}

Staphylococcus aureus and its resistant form, methicillin-resistant $S$. aureus (MRSA), rank top among the clinically most relevant nosocomial pathogens, leading to increased morbidity, mortality, length of hospital stay and costs [1-4]. Colonized healthcare workers (HCWs) are capable of developing clinical $S$. aureus infections $[5,6]$, transmitting $S$. aureus to

\footnotetext{
* Author for correspondence: K. Olsen, M.D., Department of Microbiology and Infection Control, University Hospital of North Norway, N-9038 Tromsø, Norway. (Email: karina.olsen@unn.no)
}

patients [6-8], and introducing $S$. aureus into their families [9-11]. Understanding $S$. aureus colonization patterns in HCWs may aid in the development and reinforcement of infection control strategies and add to the understanding of $S$. aureus epidemiology.

Interestingly, recent reports have revealed higher $S$. aureus nasal carriage rates in surgeons than in highrisk patient groups [12] and more frequent MRSA nasal carriage in nurses than other HCWs [13, 14], supporting the view that healthcare service may be a risk factor for carriage. However, studies in the healthcare setting generally lack a broader perceptive on the ecological context of HCWs in the community 
and how background prevalence in the general population and households may bias the results. Importantly, $S$. aureus nasal carriers may 'impose' their carrier status upon other household members $[10,11,15]$, and the bacterium can be reintroduced into the hospital by intrafamilial spread from and to HCWs $[9,11]$. Moreover, with novel technologies for molecular typing of $S$. aureus there are better opportunities for detailed epidemiological studies [9].

In The Tromsø Staph and Skin Study (TSSS), including 4026 adults aged 30-87 years, participating in a population-based health screening survey, we recently reported overall nasal carriage rates of $35 \%$ in men and $20 \%$ in women [16], and a heterogeneous distribution of $S$. aureus spa types by gender and age [17]. Importantly, we found overlapping population structures for $S$. aureus nasal isolates in this population-based screening and clinical $S$. aureus isolates in septic patients from the same geographical area and time period [17]. TSSS was designed to study the host-microbe-environment relationships in S. aureus carriage within an unselected general population, focusing on healthcare-associated environmental risk factors (i.e hospital stay, work in healthcare services) reported at the screening. Thus, to explore $S$. aureus epidemiology in $\mathrm{HCWs}$, we compared screening data on S. aureus nasal carriage, spa types and residing with children, for $\mathrm{HCW}$ s and non-HCWs from the working-age population, 30-69 years, participating in TSSS.

\section{METHODS}

\section{Population and study design}

The study subjects in TSSS were men and women who participated in the sixth survey of the Tromsø Study (Tromsø 6) conducted in 2007-2008. The Tromsø Study is an ongoing population-based cohort study with five previous surveys undertaken between 1974 and 2001 [18]. Based on the official population registry, residents of the municipality of Tromsø were invited to take part in the survey. In Tromsø 6 all subjects aged 40-42 and 60-87 years, a 40\% random sample aged $43-59$ years, and a $10 \%$ random sample aged 30-39 years were invited. In addition to the random samples, 295 participants were invited because they attended the second visit in the fourth survey in 1994. Invitation letters were sent randomly, avoiding selection bias during the sampling period. A total of 12984 men and women aged 30-87 years attended Tromsø 6 (attendance rate $65 \cdot 7 \%$ ). Due to lack of capacity and funding, young adult residents (aged 18-29 years) could not be invited [18].

For the study of $S$. aureus nasal carriage in a general population, we considered a more evenly distributed sampling across age groups to be most suitable and the inclusion of 4000 observations to be sufficient for analysis of host-microbe-environment relationships. Thus, in Tromsø 6, during October 2007-June 2008, all attendees aged 30-49 years $(n=1730)$ and random samples of older attendees aged 50-87 years $(n=2629$, relative distribution of birth cohorts as in the municipality) were asked to participate in TSSS, including a nasal swab culture at the screening (first visit) and a repeated nasal swab culture at a second visit within a few weeks [16]. A total of 4026 men and women aged 30-87 years (30-49 years, $n=1597$, age mean $=41 \cdot 1$, age median $=41$; $50-87$ years, $n=2429$, age $\mathrm{e}_{\text {mean }}=62 \cdot 6$, age $\mathrm{e}_{\text {median }}=61$ ) had at least one nasal swab culture taken and were included in TSSS, and of these, 2997 subjects (30-49 years, $n=1118$, age $\mathrm{e}_{\text {mean }}=41 \cdot 1$, age $\mathrm{e}_{\text {median }}=41 ; 50-87$ years, $n=1879$, age mean $=62 \cdot 4$, age median $=61$ ) had a repeated culture taken (Fig. 1). Mean interval between cultures was 33 days and for $90 \%$ of the observations the interval was $>12$ days.

For the present study of $S$. aureus epidemiology in HCWs, 37 subjects who had taken antibiotics within $24 \mathrm{~h}$ of the first or second visit, were excluded. According to the working age in Norway, 373 subjects aged $\geqslant 70$ years were excluded. The age of retirement in Norway is normally from 67 years, but may be postponed until age 70 years. In 2008, $22 \%$ of men and $14 \%$ of women aged $67-69$ years were registered in the Norwegian labour force [19]. Of the remaining subjects aged $<70$ years, a total of 217 (30-49 years, $n=116$, age $\mathrm{e}_{\text {mean }}=40 \cdot 9$, age $\mathrm{e}_{\text {median }}=41 ; 50-69$ years, $n=101$, age $_{\text {mean }}=59 \cdot 7$, age $_{\text {median }}=59$ ) with missing data on HCW status were excluded. Thus, for the present analysis 2279 participants aged 30-69 years (30-49 years, $n=961$, age $e_{\text {mean }}=41 \cdot 1$, age median $=41$; $50-69$ years, $n=1318$, age $\mathrm{e}_{\text {mean }}=58 \cdot 7$, age median $=59$ ) were included (Fig. 1).

The study was approved by the Regional Committee for Medical and Health Research Ethics, North Norway, and all attendees signed an informed consent form. Interviews and clinical examinations were performed at the screening centre by specially trained technicians according to standardized procedures. Body height and weight were measured, and body mass index (BMI) calculated $\left(\mathrm{kg} / \mathrm{m}^{2}\right)$ [18]. Two 


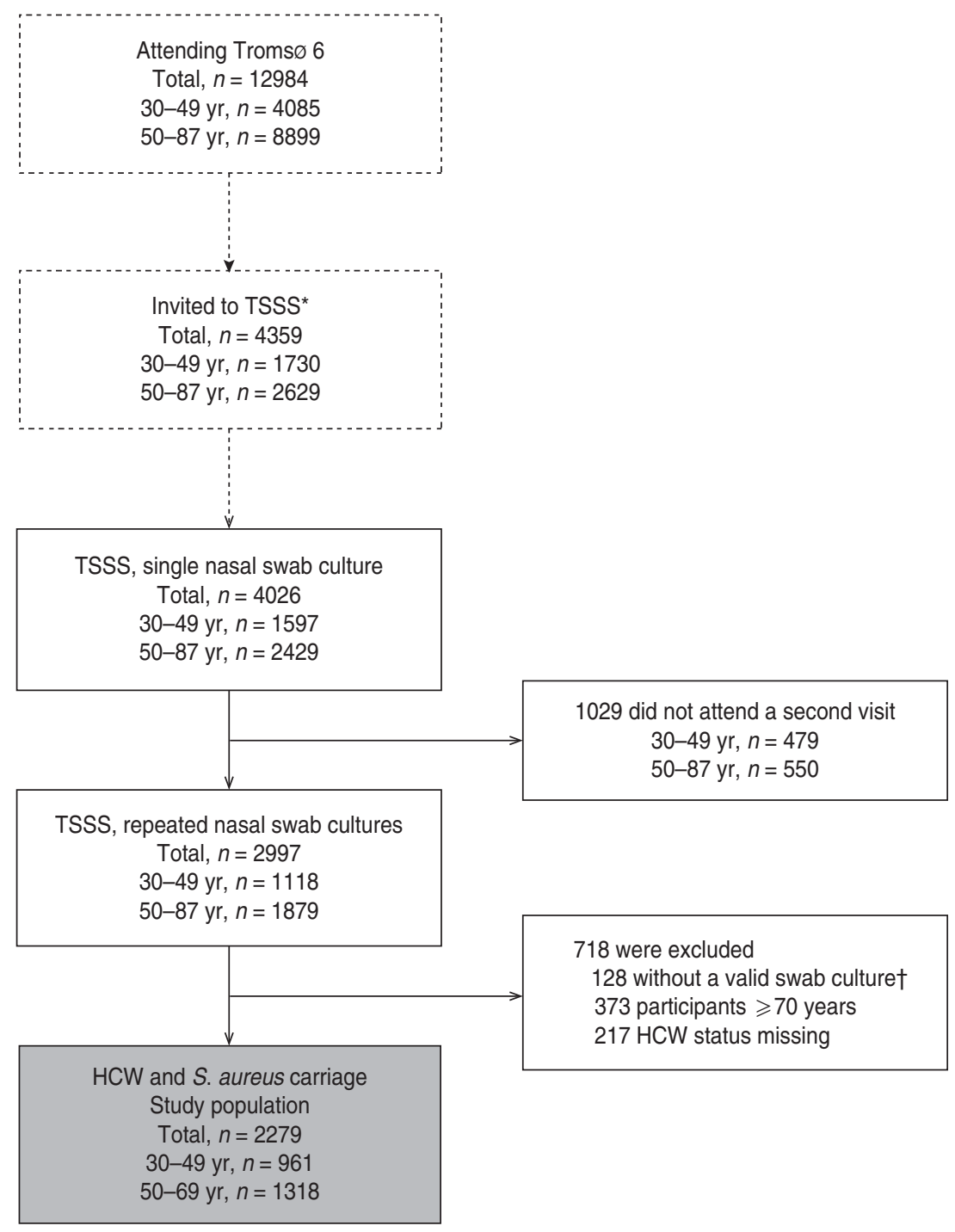

Fig. 1. The study population. TSSS, The Tromsø Staph and Skin Study; HCW, healthcare worker. * Invited to the TSSS. Age group $<50$ years: all subjects. Age group 50-87 years: random samples of subjects. $\uparrow$ Not valid swab culture: 91 had no growth in swab culture, 37 had taken antibiotics last $24 \mathrm{~h}$ before visit (systemic or eye drops/ointments).

self-administered structured questionnaires covered a broad range of issues related to health and lifestyle, including the question 'Are you residing with children aged $<18$ years?' (yes/no), referred to as 'residing with children' in the present analysis.

\section{Assessment of HCW status}

Self-reported information on current healthcare-associated environmental exposure was obtained by the interview question 'Do you work in healthcare services?'(yes/no), asked at the second visit. Healthcare services were defined as hospital, nursing home, senior care service, general practitioner (GP)'s office, and public health centre. The large-scale setting with multiple screening items in Tromsø 6 allowed for only one screening question on workplace. As an open interview question on workplace (all kinds of professions) turned out to be too time-consuming during the initial 4 weeks of the survey, it was decided to narrow it down to the specific question on work in healthcare services. This change in data collection led to missing information on HCW status until the fifth week of the survey. Thus, for the present study only the data collected after the inclusion of the specific question on work in healthcare services were analysed. The majority of HCWs in Tromsø work at the University Hospital of North Norway (UNN). UNN has about 
4400 employees and is the only hospital within a radius of $>200 \mathrm{~km}$.

\section{Detection of $S$. aureus nasal carriage}

Both vestibuli nasi were sampled by the same $\mathrm{NaCl}-$ moistened sterile rayon-tipped swab and placed in Amies charcoal transport medium (Copan, Italy). Within 3 days, all specimens were cultured at UNN, using blood agar (Oxoid, UK), chromId ${ }^{\mathrm{TM}}$ S. aureus, and chromId ${ }^{\mathrm{TM}}$ MRSA agar plates (bioMérieux, France). In order to ensure high sensitivity, all culture plates were incubated for $42-48 \mathrm{~h}$ at $37^{\circ} \mathrm{C}$. To retain high specificity, colony morphology was thoroughly examined and the most dominating suspected green colony on the chromID plates was selected for further confirmation as $S$. aureus by the Staphaurex Plus (Remel, USA) agglutination test. All confirmed $S$. aureus isolates were frozen at $-70{ }^{\circ} \mathrm{C}$ in glycerolcontaining liquid media for molecular analysis. No MRSA was isolated [16].

$S$. aureus carrier status was defined by the results of both nasal swab cultures at the first and second visits; persistent carrier $=$ two positive cultures; noncarrier $=$ one or no positive culture [20]. Our validation in 120 participants in TSSS, showed excellent inter-method reliability for $S$. aureus nasal carrier status using 1-week interval vs. 2-6 weeks between the nasal swab cultures [weighted kappa $=0 \cdot 85,95 \%$ confidence interval (CI) 0.77-0.92], also implying a low rate of transient colonization [21].

\section{spa typing}

$S$. aureus nasal isolates from the first $(99 \cdot 6 \%)$ and second $(99.9 \%)$ visits were spa typed as described elsewhere [17]. Primers recommended by Ridom GmbH (Germany) were used, i.e. spa-1113f and spa$1514 \mathrm{r}$ as described previously by Strommenger et al. [22]. Polymerase chain reaction (PCR) products were sequenced on both strands by Macrogen Korea or Macrogen Europe and spa types were assigned using Ridom StaphType software (Ridom GmbH, Germany) [23].

According to the Ridom StaphType software 264 and 268 different spa types were assigned in the first and second visits, respectively. In total, six and seven isolates were not typed due to repeated negative spa PCR amplification or deviating repeat length, respectively.

Among S. aureus nasal carriers $92.5 \%(98 / 106)$ of HCWs and $93.0 \%(453 / 487)$ of non-HCWs had the same spa type in both samples (data not shown), also in accordance with the literature [24].

\section{Statistical analysis}

Logistic regression models stratified by gender were used to estimate odds ratios (ORs) and 95\% CIs for S. aureus nasal carriage overall and by spa types associated with $\mathrm{HCW}$ status. The analysis of spa types was restricted to the nasal swab cultures of the first visit.

Selected characteristics of HCWs and non-HCWs were compared by two-sided Student's $t$ test for continuous variables and two-sided Pearson's $\chi^{2} /$ Fisher's exact test for categorical variables.

We evaluated model fit and plausibility of several covariates and the final multivariable models included age, BMI, current daily smoking (yes/no), alcohol intake ( $<2$ or $\geqslant 2$ times a week), recreational physical activity (three levels), household income ( $<$ or $\geqslant € 37000 /$ year), education level ( $<$ or $\geqslant$ college/university), and residing with children (yes/no). We tested whether residing with children modified the association between HCW status and $S$. aureus nasal carriage in stratified logistic regression analysis. Testing for interaction was done by inclusion of the multiplicative terms of the two predictor variables in the models. Tests of reliability of the final analyses were done by the Hosmer-Lemeshow goodness-of-fit test.

All tests were two-sided. Stata version 11.0 (StataCorp, USA) was used for statistical analyses.

\section{RESULTS}

HCWs comprised $334(25.7 \%)$ of 1302 women and 71 $(7 \cdot 3 \%)$ of 977 men. HCWs were younger than nonHCWs in both women and men (both $P$ values $<0.05$ ) (Table 1). Among women, HCWs were more often residing with children and reported higher household income, education level, and recreational physical activity level, and less frequent alcohol intake than non-HCWs. Among men, HCWs reported more frequent alcohol intake and hospitalization over the last 12 months than non-HCWs (all $P$ values $<0 \cdot 05$ ) (Table 1).

\section{Risk of $S$. aureus nasal carriage}

The overall prevalence of $S$. aureus nasal carriage was $26.2 \%$ in HCWs and $26.0 \%$ in non-HCWs. The 
Table 1. Characteristics of women and men by healthcare worker status. The Tromso Staph and Skin Study $\left(n=2279^{*}\right)$

\begin{tabular}{|c|c|c|c|c|c|c|}
\hline & \multicolumn{3}{|c|}{ Women $(n=1302 *)$} & \multicolumn{3}{|c|}{$\operatorname{Men}\left(n=977^{*}\right)$} \\
\hline & $\begin{array}{l}\text { Non-HCWs } \\
(n=968)\end{array}$ & $\begin{array}{l}\text { HCWs } \\
(n=334)\end{array}$ & $P \dagger$ & $\begin{array}{l}\text { Non-HCWs } \\
(n=906)\end{array}$ & $\begin{array}{l}\text { HCWs } \\
(n=71)\end{array}$ & $P \dagger$ \\
\hline Age, years & $52 \cdot 5(10 \cdot 5)$ & $49 \cdot 2(8 \cdot 9)$ & $<0 \cdot 001$ & $51 \cdot 0(9 \cdot 6)$ & $48 \cdot 1(9 \cdot 2)$ & $0 \cdot 01$ \\
\hline Higher education level $\ddagger$ & $372(38 \cdot 9)$ & $155(47 \cdot 3)$ & $0 \cdot 008$ & $386(42 \cdot 9)$ & $33(46 \cdot 5)$ & $0 \cdot 56$ \\
\hline Lower household income & $198(22 \cdot 0)$ & $43(13 \cdot 6)$ & $0 \cdot 001$ & $83(9 \cdot 4)$ & $4(5 \cdot 7)$ & $0 \cdot 30$ \\
\hline Residing with children & $339(40 \cdot 3)$ & $147(50 \cdot 9)$ & $0 \cdot 002$ & $366(45 \cdot 9)$ & $38(57 \cdot 6)$ & $0 \cdot 07$ \\
\hline Hospitalization last 12 months & $95(9 \cdot 9)$ & $33(10 \cdot 1)$ & $0 \cdot 94$ & $74(8 \cdot 2)$ & $12(17 \cdot 1)$ & $0 \cdot 01$ \\
\hline Daily smoking & $198(20 \cdot 9)$ & $70(21 \cdot 1)$ & $0 \cdot 93$ & $178(19 \cdot 8)$ & $10(14 \cdot 1)$ & $0 \cdot 24$ \\
\hline Alcohol intake $\geqslant 2$ times/week & $207(21 \cdot 6)$ & $53(16 \cdot 1)$ & $0 \cdot 03$ & $219(24 \cdot 4)$ & $25(35 \cdot 2)$ & $0 \cdot 04$ \\
\hline Low leisure physical activity\| & $179(19 \cdot 4)$ & $40(12 \cdot 5)$ & $0 \cdot 005$ & $173(19 \cdot 5)$ & $14(20 \cdot 3)$ & $0 \cdot 88$ \\
\hline Body mass index, $\mathrm{kg} / \mathrm{m}^{2}$ & $26 \cdot 5(4 \cdot 7)$ & $26 \cdot 7(5 \cdot 0)$ & $0 \cdot 41$ & $27 \cdot 6(3 \cdot 8)$ & $27 \cdot 2(3 \cdot 6)$ & 0.43 \\
\hline Diabetes & $31(3 \cdot 3)$ & $6(1 \cdot 8)$ & $0 \cdot 259$ & $25(2 \cdot 8)$ & $2(3 \cdot 0)$ & 0.719 \\
\hline Atopic eczema (ever) & $88(9 \cdot 9)$ & $36(11.9)$ & $0 \cdot 33$ & $57(6 \cdot 9)$ & $6(9 \cdot 8)$ & $0 \cdot 38$ \\
\hline
\end{tabular}

HCW, Healthcare worker.

Values are given as means (standard deviation), and numbers (\%).

* Numbers may vary due to missing information.

$\dagger$ Two-sided Student's $t$ test for continuous variables. Pearson's $\chi^{2}$ test for categorical variables.

$\ddagger \geqslant$ College/university degree.

$\S<€ 37000 /$ year.

\| Mostly sedentary recreational physical activity, e.g. watching TV.

ๆ Fisher's exact test.

corresponding sex-specific rates were $22.5 \%$ and $18 \cdot 4 \%$ in women $(P=0 \cdot 11)$, and $43 \cdot 7 \%$ and $34 \cdot 1 \%$ in men $(P=0 \cdot 10)$, respectively (Table 2$)$.

In multivariable analysis, HCW status was not associated with $S$. aureus nasal carriage in the total population (Table 2). However, female HCWs had a $54 \%$ increased risk of $S$. aureus nasal carriage $v s$. nonHCWs (OR 1.54, 95\% CI 1.09-2.19), while male HCWs did not differ from non-HCWs with respect to risk of $S$. aureus carriage.

For women residing with children, HCWs had an $86 \%$ increased risk of $S$. aureus nasal carriage compared to non-HCWs (multivariable analysis: OR $1 \cdot 86,95 \%$ CI $1 \cdot 14-3 \cdot 04 ; P$ for interaction $=0 \cdot 42$ ) (data not shown), while in women not residing with children there was no difference in risk by HCW status. For men, there was no pattern of effect modification by family status.

\section{Risk of $S$. aureus nasal carriage by spa type}

At first visit, a total of $61,41,32,26,22$ and 15 participants were colonized with spa types t012, t084, $\mathrm{t} 065, \mathrm{t} 021, \mathrm{t} 015$ and $\mathrm{t} 002$, respectively, which were the predominant spa types including almost one third of the $S$. aureus nasal isolates. The distribution of the
31 most common ( $\geqslant 4$ observations) spa types in the first sample according to HCW status is shown in Figure 2. The majority of these spa types were observed in both HCWs and non-HCWs, while seven spa types were restricted to non-HCWs, among these spa type t002. None of the most common spa types were found in HCWs only.

Among S. aureus nasal carriers, HCWs had 2.17 and $3 \cdot 16$ times higher risk of spa types t012 and t015 in the first sample, respectively, compared to non-HCWs (multivariable analysis: OR $2 \cdot 17,95 \%$ CI $1 \cdot 16-4.08$ and OR $3 \cdot 16,95 \%$ CI $1 \cdot 13-8 \cdot 87$ ) (Table 3).

When restricting the analysis to $S$. aureus nasal carriers residing with children, HCWs had a 2.42 times higher risk of spa type t012 in the first sample compared to non-HCWs (age- and gender-adjusted analysis: OR $2 \cdot 42,95 \%$ CI $1 \cdot 03-5 \cdot 70$ ). The association for spa type $\mathrm{t} 012$ was particularly strong in male nasal carriers (age-adjusted analysis: OR 4.61, $95 \%$ CI 1.36-15.61) (Table 4). Among S. aureus nasal carriers not residing with children, $\mathrm{HCW}$ s had a fourfold increased risk of spa type t015 in the first sample compared to non-HCWs (age- and genderadjusted analysis: OR $4 \cdot 28,95 \%$ CI $0.99-18 \cdot 43$ ) (Table 4). 
Table 2. Estimated risk of $\mathrm{S}$. aureus nasal carriage by healthcare worker status. Logistic regression analysis. The Tromso Staph and Skin Study $(n=2279 *)$

\begin{tabular}{lcccccc}
\hline \hline & $\begin{array}{l}\text { Total } \\
\text { population }\end{array}$ & Carriers $(\%)$ & OR $\dagger$ & $95 \%$ CI & OR $\dagger$ & $95 \%$ CI \\
\hline Both genders & & & & & & \\
$\quad$ Non-HCWs & 1874 & $487(26 \cdot 0)$ & Ref. & & Ref. & \\
$\quad$ HCWs & 405 & $106(26 \cdot 2)$ & $0 \cdot 98$ & $(0 \cdot 77-1 \cdot 26)$ & $1 \cdot 08$ & $(0 \cdot 82-1 \cdot 41)$ \\
Women & & $178(18 \cdot 4)$ & Ref. & & Ref. & \\
$\quad$ Non-HCWs & 968 & $75(22 \cdot 5)$ & $1 \cdot 29$ & $(0 \cdot 95-1 \cdot 76)$ & $1 \cdot 54$ & $(1 \cdot 09-2 \cdot 19)$ \\
$\quad$ HCWs & 334 & $309(34 \cdot 1)$ & Ref. & & Ref. & \\
Men & & $31(43 \cdot 7)$ & $1 \cdot 44$ & $(0 \cdot 88-2 \cdot 35)$ & $1 \cdot 45$ & $(0 \cdot 86-2 \cdot 45)$ \\
$\quad$ Non-HCWs & 906 & 71 & & &
\end{tabular}

HCW, Healthcare worker; OR, odds ratio; CI, confidence interval.

* Numbers may vary due to missing information.

$\dagger$ Age-adjusted.

\# Multivariable logistic regression model including: age, current daily smoking (yes/no), alcohol intake ( $<2$ or $\geqslant 2$ times a week), recreational physical activity (three levels), household income ( $<$ or $\geqslant € 37000 /$ year), residing with children (yes/no), body mass index, education level ( $<$ or $\geqslant$ college/university degree).

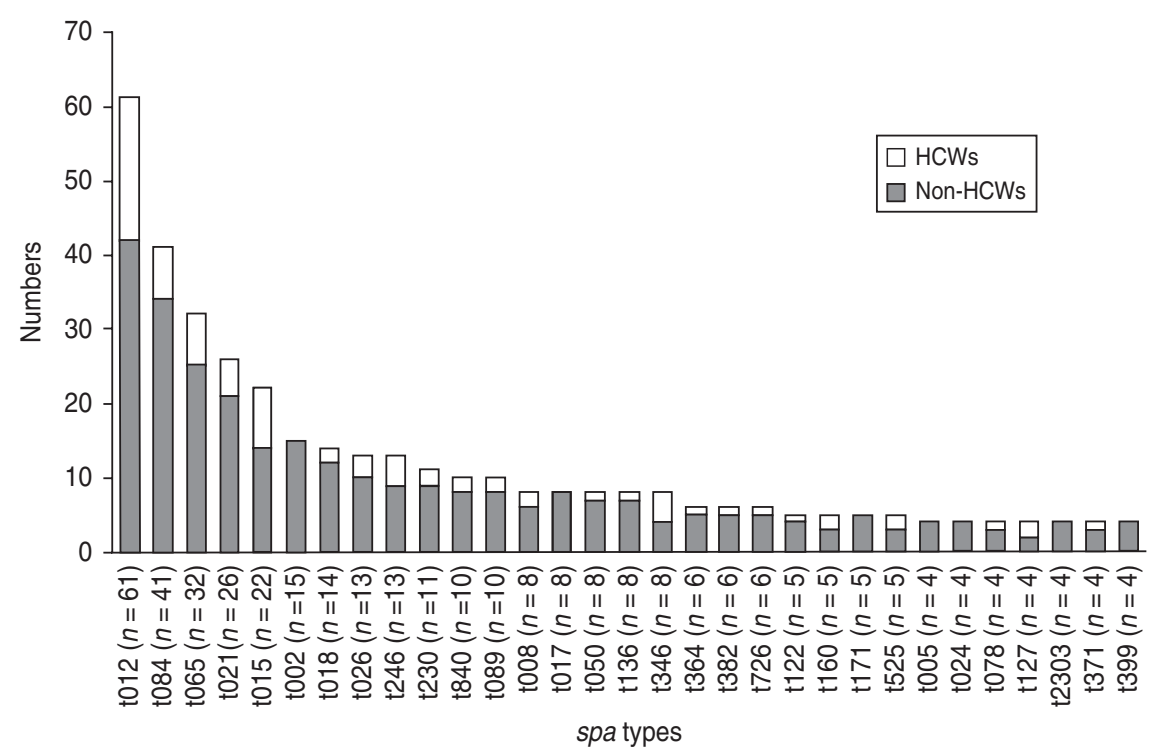

Fig. 2. Distribution of spa types in non-healthcare workers (non-HCWs) and healthcare workers (HCWs) at first visit, nasal swab cultures. The Tromsø Staph and Skin Study. spa types with $\leqslant 3$ observations are not shown (233 different spa types in 298 participants).

\section{DISCUSSION}

In this large population-based study with participants aged 30-69 years with repeated nasal swab cultures, working in healthcare services was associated with a $54 \%$ increased risk of $S$. aureus nasal carriage in women. The risk was even higher in women residing with children. For men, work in healthcare services and residing with children were associated with increased prevalence of common spa types. Our study suggests that a synergism between environmental risk factors (work and household) is of importance for $S$. aureus carrier status in HCWs.

To our knowledge, this is the first study which reports that female $\mathrm{HCWs}$ have a higher risk of $S$. aureus nasal carriage than female non-HCWs in a general working-age population. Interestingly, other studies confined to HCWs, observed higher nasal carriage rates of MRSA in nurses than in other healthcare professionals, and cited increased patient 
Table 3. Estimated risk of the six most common spa types at first sample by healthcare worker status. S. aureus nasal carriers $(n=593)$, The Tromsø Staph and Skin Study

\begin{tabular}{|c|c|c|c|c|c|c|}
\hline & \multirow{2}{*}{$\begin{array}{l}\begin{array}{l}\text { Non-HCWs } \\
(n=487)\end{array} \\
n(\%)\end{array}$} & \multirow{2}{*}{$\begin{array}{l}\begin{array}{l}\mathrm{HCWs} \\
(n=106)\end{array} \\
n(\%)\end{array}$} & \multirow[b]{2}{*}{ OR* } & \multirow[b]{2}{*}{$95 \% \mathrm{CI}$} & \multirow[b]{2}{*}{$\mathrm{OR} \dagger$} & \multirow[b]{2}{*}{$95 \% \mathrm{CI}$} \\
\hline & & & & & & \\
\hline $\mathrm{t} 012(n=57)$ & $38(7 \cdot 8)$ & $19(17 \cdot 9)$ & $2 \cdot 58$ & $(1 \cdot 42-4 \cdot 69)$ & $2 \cdot 17$ & $(1 \cdot 16-4 \cdot 08)$ \\
\hline $\mathrm{t} 084(n=31)$ & $27(5 \cdot 5)$ & $4(3 \cdot 8)$ & 0.67 & $(0 \cdot 23-1 \cdot 95)$ & $0 \cdot 74$ & $(0 \cdot 24-2 \cdot 23)$ \\
\hline $\mathrm{t} 065(n=27)$ & $20(4 \cdot 1)$ & $7(6 \cdot 6)$ & $1 \cdot 65$ & $(0 \cdot 68-4 \cdot 01)$ & 1.94 & $(0 \cdot 75-4 \cdot 98)$ \\
\hline $\mathrm{t} 021(n=20)$ & $16(3 \cdot 3)$ & $4(3 \cdot 8)$ & $1 \cdot 15$ & $(0 \cdot 38-3 \cdot 53)$ & $1 \cdot 48$ & $(0 \cdot 45-4 \cdot 79)$ \\
\hline $\mathrm{t} 015(n=19)$ & $12(2 \cdot 5)$ & $7(6 \cdot 6)$ & $2 \cdot 80$ & $(1 \cdot 07-7 \cdot 29)$ & $3 \cdot 16$ & $(1 \cdot 13-8 \cdot 87)$ \\
\hline t002 $(n=12) \ddagger$ & $12(2 \cdot 5)$ & $0(0 \cdot 0)$ & & & & \\
\hline
\end{tabular}

HCW, Healthcare worker; OR, odds ratio; CI, confidence interval.

* Logistic regression model (unadjusted).

$\uparrow$ Multivariable logistic regression model including: age and gender.

$\ddagger$ No OR estimates due to no spa type t002 in HCWs.

Table 4. Estimated risk of spa types t012 and t015 nasal carriage by healthcare worker status and residing with children. Logistic regression analysis. The Tromsø Staph and Skin Study

\begin{tabular}{|c|c|c|c|c|c|c|c|c|}
\hline & \multicolumn{4}{|c|}{ Residing with children $(n=244)$} & \multicolumn{4}{|c|}{ Not residing with children $(n=279)$} \\
\hline & Non-HCWs & $\mathrm{HCWs}$ & & & Non-HCWs & HCWs & & \\
\hline & $n(\%)$ & $n(\%)$ & OR & $95 \% \mathrm{CI}$ & $n(\%)$ & $n(\%)$ & OR & $95 \% \mathrm{CI}$ \\
\hline \multicolumn{9}{|l|}{$\mathrm{t} 012$} \\
\hline Both genders & $18(9 \cdot 6)$ & $12(21 \cdot 4)$ & $2 \cdot 42 *$ & $(1 \cdot 03-5 \cdot 70)$ & $18(7 \cdot 6)$ & $6(14 \cdot 0)$ & $1 \cdot 41 *$ & $(0 \cdot 50-3 \cdot 96)$ \\
\hline Women & $8(13 \cdot 6)$ & $7(18 \cdot 4)$ & $1 \cdot 61 \dagger$ & $(0 \cdot 52-5 \cdot 02)$ & $9(10 \cdot 0)$ & $6(19 \cdot 4)$ & $1 \cdot 89 \dagger$ & $(0 \cdot 60-5 \cdot 93)$ \\
\hline Men $\ddagger$ & $10(7 \cdot 8)$ & $5(27 \cdot 8)$ & $4 \cdot 61 \dagger$ & $(1 \cdot 36-15 \cdot 61)$ & $9(6 \cdot 6)$ & 0 & & \\
\hline \multicolumn{9}{|l|}{$\mathrm{t} 015$} \\
\hline Both genders & $4(2 \cdot 1)$ & $3(5 \cdot 4)$ & $3 \cdot 61 *$ & $(0 \cdot 70-18 \cdot 67)$ & $5(2 \cdot 1)$ & $4(9 \cdot 3)$ & $4 \cdot 28 *$ & $(0 \cdot 99-18 \cdot 43)$ \\
\hline
\end{tabular}

HCW, Healthcare worker; OR, odds ratio; CI, confidence interval.

* Multivariable logistic regression model adjusted for age and gender.

$\dagger$ Multivariable logistic regression model adjusted for age.

\& No OR estimate due to no spa type t012 in male HCWs not residing with children.

contact as a cause $[13,14]$. Our screening study did not include information about the HCWs' profession. However, the majority of the Norwegian healthcare workforce are nurses and auxiliary nurses, and about $90 \%$ of these are women $[25,26]$. Thus, our findings suggest that work in healthcare services is an environmental risk factor for $S$. aureus nasal carriage in women, due to a substantial amount of contact with patients and the elderly in residential care when working as nurses and auxiliary nurses. No MRSA was isolated in our study. However, epidemiological evidence supports a similar mechanism for transmission of methicillin-sensitive S. aureus (MSSA) and MRSA usually via direct contact with patients and other close contacts (household members, etc.) $[27,28]$.
We observed that working in healthcare services in combination with residing with children was a high risk cluster for $S$. aureus carriage in women. Our findings are in agreement with current literature supporting the view that contact transmission within HCWs' families may affect the burden of MSSA and MRSA infections in hospitals [9, 11]. Importantly, residing with children per se was not associated with $S$. aureus nasal carriage in our study, and number of children aged $<18$ years and age did not differ between HCWs and non-HCWs (results not shown). Information on childcare was not included in our study, and attending kindergarten has been associated with increased risk of $S$. aureus colonization in children [29]. However, it can be assumed that use of kindergarten was not a major confounder in our 
analysis, as there have been places in kindergarten for all children in Tromsø since 2005 [30] and 89.4\% of female non-HCWs residing with children were part-time or full-time workers. Thus, we hypothesize that the environmental pressure caused by high rates of contact transmission of $S$. aureus from both patients and children may exceed the ability of female HCWs (i.e through hand hygiene, immune responses) to defend themselves against colonization.

The population structure of $S$. aureus colonizing HCWs and non-HCWs was similar, as expected from others [13] and our previous comparative study of clinical and nasal carrier $S$. aureus isolates in Tromsø [17]. However, we observed that work in healthcare services was associated with an increased risk of spa types $\mathrm{t} 012$ and $\mathrm{t} 015$, which both ranked in the top five in our study and are also frequent worldwide [31, 32]. Thus, we may expect spa types t012 and t015 to be frequent causes of autoinfections in patients in the community and in hospitals, with an increased potential for transmission, that may also partly explain our findings.

Among men, HCW status and residing with children predicted $S$. aureus carriage by spa types and not overall carriage rates. Even though there is considerable degree of uncertainty in our estimates, we can hypothesize that in the male human population, common $S$. aureus spa types have specific main niches defined by environmental factors. The female preference of the common spa type t012 in our study population, may possibly confound the results in women [17].

The main limitation of our study is the lack of information on profession, workplace and patient contact. The cross-sectional design demands caution in interpretation regarding any causal relationship. Thus, future prospective population-based studies including work and home exposures are needed to improve our knowledge about high-risk groups and environmental targets for more effective prevention of $S$. aureus carriage in HCWs. As the prevalence of $S$. aureus carriage in younger adults is relatively high, particularly in men [33,34], the role of work in healthcare services for carriage should also be explored in the working population aged $<30$ years. Furthermore, the S. aureus epidemiology in HCWs having contact with the elderly requires increased focus given the fact that nursing-home residents are a population at risk for carrying S. aureus and MRSA [35]. Recent antibiotic use not registered at the screening (i.e. ceased $>24 \mathrm{~h}$ before visit) and lack of enrichment during culturing may have caused false negatives independently of HCW status. Thus, nondifferential misclassification of $S$. aureus nasal carrier status may have biased our odds ratio estimates towards null.

The major strengths of our study are the population-based design, high participation rate, large set of observations, validated outcome variable including spa type, gender-stratified analysis, and the adjustment for major confounders (i.e. age, BMI, smoking, etc.). Furthermore, representative data were achieved as demonstrated by the age distribution within the different age groups included.

The absence of MRSA in this study is in line with the low prevalence of MRSA in clinical S. aureus samples registered in the Norwegian surveillance programme during 2007-2008 [36]. Importantly, the surveillance data do not reflect a healthy population, and therefore cannot be directly compared to MRSA/ MSSA colonization rates in our study, including healthy persons only (i.e not hospitalized or institutionalized).

In conclusion, our study indicates that work in healthcare services in combination with residing with children is a high-risk cluster for $S$. aureus carriage in women. The synergism of work and home environmental risk factors should be addressed in future studies to increase our understanding of $S$. aureus epidemiology in HCWs and suggest novel targets for improved infection control strategies against exogenous MSSA and MRSA infections in patients.

\section{ACKNOWLEDGEMENTS}

We thank Bjørg C. Haldorsen, Trine Tessem, Bettina Aasnæs and Tonje Holan for excellent technical assistance. This work was supported by grants from the Research Council of Norway (grant no. 191264); Northern Norway Regional Health Authority (project no. 7150.00003, grant SFP877-09 and SFP920-10); and the Odd Berg Group Medical Research fund 2007.

\section{DECLARATION OF INTEREST}

None.

\section{REFERENCES}

1. Diekema DJ, et al. Survey of infections due to Staphylococcus species: frequency of occurrence and 
antimicrobial susceptibility of isolates collected in the United States, Canada, Latin America, Europe, and the Western Pacific region for the SENTRY Antimicrobial Surveillance Program, 1997-1999. Clinical Infectious Diseases 2001; 32 (Suppl 2): 114-132.

2. Kirkland KB, et al. The impact of surgical-site infections in the 1990s: attributable mortality, excess length of hospitalization, and extra costs. Infection Control Hospital Epidemiology 1999; 20: 725-730.

3. Kock R, et al. Methicillin-resistant Staphylococcus aureus (MRSA): burden of disease and control challenges in Europe. Eurosurveillance 2010; 15: 19688.

4. Pittet D, Wenzel RP. Nosocomial bloodstream infections. Secular trends in rates, mortality, and contribution to total hospital deaths. Archives of Internal Medicine 1995; 155: 1177-1184.

5. von Eiff C, et al. Nasal carriage as a source of Staphylococcus aureus bacteremia. Study Group. New England Journal of Medicine 2001 ; 344: 11-16.

6. Wertheim HF, et al. Risk and outcome of nosocomial Staphylococcus aureus bacteraemia in nasal carriers versus non-carriers. Lancet 2004; 364: 703-705.

7. Blok HE, et al. Role of healthcare workers in outbreaks of methicillin-resistant Staphylococcus aureus: a 10-year evaluation from a Dutch university hospital. Infection Control Hospital Epidemiology 2003; 24: 679-685.

8. Tammelin A, et al. Nasal and hand carriage of Staphylococcus aureus in staff at a Department for Thoracic and Cardiovascular Surgery: endogenous or exogenous source? Infection Control Hospital Epidemiology 2003; 24: 686-689.

9. Lu PL, et al. Methicillin-resistant Staphylococcus aureus carriage, infection and transmission in dialysis patients, healthcare workers and their family members. Nephrology Dialysis Transplantation 2008; 23: 1659-1665.

10. Mollema FP, et al. Transmission of methicillin-resistant Staphylococcus aureus to household contacts. Journal of Clinical Microbiology 2010; 48: 202-207.

11. Wagenvoort JH, et al. Risk of re-introduction of methicillin-resistant Staphylococcus aureus into the hospital by intrafamilial spread from and to healthcare workers. Journal of Hospital Infection 2005; 59: 67-68.

12. Schwarzkopf R, et al. Prevalence of Staphylococcus aureus colonization in orthopaedic surgeons and their patients: a prospective cohort controlled study. Journal of Bone \& Joint Surgery 2010; 92: 1815-1819.

13. Elie-Turenne MC, et al. Prevalence and characteristics of Staphylococcus aureus colonization among healthcare professionals in an urban teaching hospital. Infection Control Hospital Epidemiology 2010; 31: 574-580.

14. Suffoletto BP, et al. Prevalence of Staphylococcus aureus nasal colonization in emergency department personnel. Annals of Emergency Medicine 2008; 52: 529-533.

15. Peacock SJ, et al. Determinants of acquisition and carriage of Staphylococcus aureus in infancy. Journal of Clinical Microbiology 2003; 41 : 5718-5725.
16. Olsen K, et al. Staphylococcus aureus nasal carriage is associated with serum 25-hydroxyvitamin D levels, gender and smoking status. The Tromsø Staph and Skin Study. European Journal of Clinical Microbiology \& Infectious Diseases. Published online: 3 August 2011; doi: 10.1007/s10096-011-1331-x.

17. Sangvik M, et al. Age- and gender-associated Staphylococcus aureus spa types found among nasal carriers in a general population: the Tromso Staph and Skin Study. Journal of Clinical Microbiology 2011; 49: 4213-4218.

18. Jacobsen BK, et al. Cohort profile: The Tromsø Study. International Journal of Epidemiology. Published online: 21 March 2011; doi: 10.1093/ije/dyr049.

19. Statistics Norway. Statistical Yearbook of Norway 2008. Labour Market (http://www.ssbno), 2009, pp. 205-229.

20. van Belkum A, et al. Reclassification of Staphylococcus aureus nasal carriage types. Journal of Infectious Diseases 2009; 199: 1820-1826.

21. Haldorsen B, et al. Nasal and throat swab cultures for the assessment of Staphylococcus aureus colonization and carriage. The Tromsø Staph and Skin Study. European Congress of Clinical Microbiology and Infectious Diseases (ECCMID), 2009. Helsinki. Abstract number 1150 .

22. Strommenger B, et al. spa typing of Staphylococcus aureus as a frontline tool in epidemiological typing. Journal of Clinical Microbiology 2008; 46: 574-581.

23. Harmsen D, et al. Typing of methicillin-resistant Staphylococcus aureus in a university hospital setting by using novel software for spa repeat determination and database management. Journal of Clinical Microbiology $2003 ; 41$ : 5442-5448.

24. Sakwinska O, et al. Ecological temporal stability of Staphylococcus aureus nasal carriage. Journal of Clinical Microbiology 2010; 48: 2724-2728.

25. Køber T, Vigran A. Samfunnsspeilet no. 2, 2011 [in Norwegian]. Statistics Norway (http://www.ssbno/), 2011.

26. Statistics Norway. Increase in health workers -2010 (http://www.ssbno/english/subjects/06/01/hesospers_en/), 2011.

27. Calfee DP. The epidemiology, treatment, and prevention of transmission of methicillin-resistant Staphylococcus aureus. Journal of Infusion Nursing 2011; 34: 359-364.

28. Skov R, et al. Update on the prevention and control of community-acquired meticillin-resistant Staphylococcus aureus (CA-MRSA). International Journal of Antimicrobial Agents. Published online: 9 January 2012; doi: 10.1016/j.ijantimicag.2011.09.029.

29. Lamaro-Cardoso J, et al. Molecular epidemiology and risk factors for nasal carriage of Staphylococcus aureus and methicillin-resistant $S$. aureus in infants attending day care centers in Brazil. Journal of Clinical Microbiology 2009; 47: 3991-3997.

30. Statistics Norway. Statistical Yearbook of Norway 2008. Education (http://www.ssbno) 2009; pp. 157-188. 
31. Fenner L, et al. Molecular epidemiology of invasive methicillin-susceptible Staphylococcus aureus strains circulating at a Swiss University Hospital. European Journal of Clinical Microbiology \& Infectious Diseases 2008; 27 : 623-626.

32. Grundmann H, et al. Geographic distribution of Staphylococcus aureus causing invasive infections in Europe: a molecular-epidemiological analysis. PLoS Medicine 2010; 7 : e1000215.

33. Gorwitz RJ, et al. Changes in the prevalence of nasal colonization with Staphylococcus aureus in the United States, 2001-2004. Journal of Infectious Diseases 2008; 197: 1226-1234.
34. Olsen K, et al. The epidemiology of Staphylococcus aureus nasal and throat carriage in a large communitybased population in Northern Norway. The Tromsø Staph and Skin Study. European Congress of Clinical Microbiology and Infectious Diseases (ECCMID) 2009. Helsinki. Abstract number 1678.

35. Pfingsten-Wurzburg S, et al. Prevalence and molecular epidemiology of meticillin-resistant Staphylococcus aureus in nursing home residents in northern Germany. Journal of Hospital Infection 2011; 78: 108-112.

36. NORM/NORM-VET 2008. Usage of antimicrobial agents and occurence of antimicrobial resistance in Norway. Tromsø/Oslo, 2009. 